\title{
Pacific
}

Journal of

Mathematics

\section{GEOMETRIC FORMALITY \\ OF HOMOGENEOUS SPACES AND OF BIQUOTIENTS}

D. KotsChICK AND S. TERZIĆ 


\title{
GEOMETRIC FORMALITY OF HOMOGENEOUS SPACES AND OF BIQUOTIENTS
}

\author{
D. KOTSCHICK AND S. TERZIĆ
}

\begin{abstract}
We provide examples of homogeneous spaces that are neither symmetric spaces nor real cohomology spheres, yet have the property that every invariant metric is geometrically formal. We also extend the known obstructions to geometric formality to some new classes of homogeneous spaces and of biquotients, and to certain sphere bundles.
\end{abstract}

\section{Introduction}

The notion of geometric formality was implicitly considered by Sullivan in the 1970s (see [Deligne et al. 1975; Sullivan 1975]), but the systematic study of this concept began only comparatively recently [Kotschick 2001]. A smooth manifold is geometrically formal if it admits a Riemannian metric for which all exterior products of harmonic forms are harmonic. Such a metric is then also called formal. Geometric formality clearly implies formality in the sense of Sullivan, and is even more restrictive. Since compact symmetric spaces are the classical examples of geometrically formal manifolds, it is natural to explore this notion in the context of homogeneous spaces, or, more generally, of manifolds with large symmetry groups.

Trying to come up with generalizations of symmetric spaces, one might think first of isotropy irreducible spaces. These are the homogeneous spaces $G / H$ for which the isotropy representation of $H$ on $T_{e H}(G / H)$ is irreducible. Such a space is strongly isotropy irreducible if the restriction of the isotropy representation to the identity component of $H$ is also irreducible. These manifolds were originally classified by Manturov, and were further studied by Wolf and others, cf. [Besse 1987]. They share many properties of symmetric spaces, and indeed irreducible symmetric spaces are isotropy irreducible. A conceptual relationship between symmetric spaces and isotropy irreducible ones is explained in [Wang and Ziller 1993].

MSC2000: primary 53C25, 53C30; secondary 57T15, 57T20, 58A 10.

Keywords: formality, harmonic forms, homogeneous space, Stiefel manifold, biquotient.

The work of the first author was supported in part by the DFG Priority Program in Global Differential Geometry and by The Bell Companies Fellowship at the Institute for Advanced Study in Princeton. Copyright (c) 2009 D. Kotschick and S. Terzić. 
However, the similarities between symmetric spaces and isotropy irreducible ones do not extend to (geometric) formality. Indeed, there are a number of strongly isotropy irreducible spaces which, by the results of [Kuin' 1968], are not of Cartan type and therefore [Kotschick and Terzić 2003] are not formal in the sense of Sullivan. A fortiori, they cannot be geometrically formal.

Example 1. The compact homogeneous spaces $\mathrm{SU}(p q) /(\mathrm{SU}(p) \times \mathrm{SU}(q))$ for $p, q \geq 3, \quad \mathrm{SO}(78) / E_{6}$, and $\mathrm{SO}\left(n^{2}-1\right) / \mathrm{SU}(n)$ for $n \geq 3$ are strongly isotropy irreducible, but are not formal in the sense of Sullivan.

Another class of homogeneous spaces generalizing the symmetric ones consists of the so-called generalized symmetric spaces, sometimes called $k$-symmetric spaces. These are defined by replacing the involution in the definition of symmetric spaces by a symmetry of order $k$; see [Wolf and Gray 1968a; 1968b; Terzich 2001]. In [Kotschick and Terzić 2003] we proved that all generalized symmetric spaces of compact simple Lie groups are formal in the sense of Sullivan, and that many of them are not geometrically formal.

The main purpose of this paper is to prove that, in spite of all these negative results, there are homogeneous spaces that are neither (homotopy equivalent to) symmetric spaces nor products of real homology spheres (which are trivially geometrically formal [Kotschick 2001]), yet are geometrically formal. We shall prove the following:

Theorem 2. All homogeneous metrics on the following homogeneous spaces are geometrically formal:

(1) the real Stiefel manifolds $V_{4}\left(\mathbb{R}^{2 n+1}\right)=\mathrm{SO}(2 n+1) / \mathrm{SO}(2 n-3)$ for $n \geq 3$,

(2) the real Stiefel manifolds $V_{3}\left(\mathbb{R}^{2 n}\right)=\mathrm{SO}(2 n) / \mathrm{SO}(2 n-3)$ for $n \geq 3$,

(3) the complex Stiefel manifolds $V_{2}\left(\mathbb{C}^{n}\right)=\mathrm{SU}(n) / \mathrm{SU}(n-2)$ for $n \geq 5$,

(4) the quaternionic Stiefel manifolds $V_{2}\left(\mathbb{M}^{n}\right)=\operatorname{Sp}(n) / \mathrm{Sp}(n-2)$ for $n \geq 3$,

(5) the octonionic Stiefel manifold $V_{2}\left(\mathbb{O}^{2}\right)=\operatorname{Spin}(9) / G_{2}$, and

(6) the space $\operatorname{Spin}(10) / \operatorname{Spin}(7)$.

Moreover, none of these spaces is homotopy equivalent to a symmetric space. They are not homotopy equivalent to products of real cohomology spheres, except possibly for $V_{3}\left(\mathbb{R}^{2 n}\right)$ with $n$ even.

The space $\operatorname{Spin}(10) / \operatorname{Spin}(7)$ above corresponds to a nonstandard embedding of $\operatorname{Spin}(7)$ in $\operatorname{Spin}(10)$, so that the quotient is not $V_{3}\left(\mathbb{R}^{10}\right)$ but a manifold with the same real homology and different integral homology. The homology of this space, unlike that of $V_{3}\left(\mathbb{R}^{10}\right)$, is torsion-free.

In Section 2, we exhibit a very simple mechanism to prove that $G$-invariant metrics on certain homogeneous spaces $G / H$ with simple cohomology rings are 
geometrically formal. This mechanism in fact gives a new proof that certain symmetric spaces are geometrically formal, without using the symmetric space structure, but only the description of the cohomology ring. The argument applies to all homogeneous spaces that have the real cohomology of a product of odddimensional spheres. The examples (3)-(6) in Theorem 2 are all the homogeneous spaces that have the integral cohomology of such a product, but are not obviously diffeomorphic to products or to symmetric spaces. There are many more examples with the real cohomology of such a product, but different integral cohomology. We discuss the two infinite sequences of real Stiefel manifolds occurring in (1) and (2), leaving aside the other, sporadic, examples. Also in Section 2, by considering the Aloff-Wallach spaces [Aloff and Wallach 1975], we show that our results do not extend to all homogeneous spaces with the cohomology algebra of a product of spheres, if one does not insist that the spheres be odd-dimensional. In Section 3 we show that the homogeneous spaces listed in Theorem 2 are not homotopy equivalent to symmetric spaces or to nontrivial products, thereby completing the proof of the theorem.

In the final two sections of this paper we add to the negative results of [Kotschick 2001; Kotschick and Terzić 2003; 2009] by providing further examples of manifolds that, though formal in the sense of Sullivan, are not geometrically formal. All the examples we give here are simply connected and of dimension six. In Section 4 we consider certain classes of biquotients in the sense of Eschenburg [1992b; 1992a] and in Section 5 we consider two-sphere bundles over $\mathbb{C} P^{2}$. Many of these two-sphere bundles are known to carry special metrics of cohomogeneity one by the results of [Grove and Ziller 2008]. Both these collections of examples generalize the discussion of the flag manifold $\mathrm{SU}(3) / T^{2}$ carried out in [Kotschick and Terzić 2003]. A different generalization, to certain partial flag manifolds of higher dimensions, is contained in [Kotschick and Terzić 2009].

\section{Examples of geometrically formal homogeneous spaces}

In this section, we describe a class of homogeneous spaces for which any homogeneous metric is formal.

First, we recall the well-known fact that, on a compact homogeneous space $G / H$, the harmonic forms of any homogeneous metric are invariant. To check this, let $h$ be a harmonic form with respect to some homogeneous metric on $G / H$. Then $h$ and $* h$ can be written as $h=h_{i}+d \alpha$, and $* h=(* h)_{i}+d \beta$, where $h_{i}=\int_{G} g^{*} h$ and $(* h)_{i}=\int_{G} g^{*}(* h)$ with respect to Haar measure with total volume 1 . Since $h_{i}$ and $(* h)_{i}$ are invariant forms, we have

$$
* h_{i}=* \int_{G} g^{*} h=\int_{G} *\left(g^{*} h\right)=\int_{G} g^{*}(* h)=(* h)_{i} .
$$


It follows that $d\left(* h_{i}\right)=d(* h)_{i}=0$ and thus $h_{i}$ is harmonic. This means that $h=h_{i}$, that is, $h$ is an invariant form.

As a first application of this fact, we have the following:

Proposition 3. If $G$ is a compact connected Lie group and $H$ is a closed connected subgroup with the property that $G / H$ is of even dimension $2 k$, and all its real cohomology is in degrees $0, k$ and $2 k$, then any homogeneous metric on $G / H$ is formal.

Proof. Because of the cohomology structure of $G / H$, to prove that it is geometrically formal with respect to some metric, it is enough to prove that $x \wedge y$ is a harmonic form for any two harmonic $k$-forms $x$ and $y$. If the metric $g$ is homogeneous, then according to the previous observation, $x \wedge y$ is an invariant form and being of top degree, we have $x \wedge y=c \cdot d v o l$, where $c$ is constant. Thus, $x \wedge y$ is harmonic and $g$ is formal.

Example 4. The complex projective plane $\mathbb{C} P^{2}=\mathrm{SU}(3) / \mathrm{S}(\mathrm{U}(2) \times \mathrm{U}(1))$, the quaternionic projective plane $\mathbb{H} P^{2}=\mathrm{Sp}(3) /(\mathrm{Sp}(2) \times \mathrm{Sp}(1))$, the Cayley plane $\mathbb{O} P^{2}=F_{4} / \operatorname{Spin}(9)$, and $G_{2} / \mathrm{SO}(4)$ are examples to which the proposition applies; cf. [Borel and Hirzebruch 1958].

These spaces are all symmetric, but the argument proving geometric formality does not use the symmetric space structure. Unfortunately, there are no nonsymmetric homogeneous spaces to which we could apply Proposition 3. Indeed, if the number $k$ is even, then such spaces belong to the class of rank one homogeneous spaces in the terminology of Onishchik [1995], while for odd $k$ they belong to the class of rank two homogeneous spaces. Examining the classification of homogeneous spaces of rank one and two given by Onishchik [1995], one sees that there are no examples other than the symmetric spaces mentioned in Example 4.

To get new examples, we need the following slight variation of Proposition 3:

Proposition 5. If $G$ is a compact connected Lie group and $H$ is a closed connected subgroup with the property that $H^{*}(G / H ; \mathbb{R})=\bigwedge(x, y)$, where $x$ and $y$ are of odd degrees, then any homogeneous metric on $G / H$ is formal.

The spaces listed in Theorem 2 all have cohomology rings of this form; cf. [Onishchik 1963; Kramer 2002]. Thus Proposition 5 shows that those spaces are geometrically formal.

Proof. Because of the cohomology structure of $G / H$, to prove that it is geometrically formal with respect to some metric, it is enough to prove that $x \wedge y$ is a harmonic form, where $x$ and $y$ are the harmonic representatives of the cohomology generators. If the metric $g$ is homogeneous, then as before, $x \wedge y$ is an invariant form and, being of top degree, we have $x \wedge y=c \cdot d v o l$, where $c$ is constant. It follows that $x \wedge y$ is harmonic and $g$ is formal. 
Remark 6. At this point it is useful to recall that if $G$ is simple and we endow $G / H$ with the submersion metric $g$ of a biinvariant metric on $G$, then the Riemannian homogeneous space $(G / H, g)$ is irreducible as a Riemannian manifold [Kobayashi and Nomizu 1963]. This means that such a $g$ cannot be a product metric. These are examples of normal homogeneous metrics.

As a simple application of our discussion so far, we can prove the following:

Proposition 7. The group SU(4) acts transitively on $S^{5} \times S^{7}$. All SU(4)-homogeneous metrics for this action are formal. Furthermore, the normal homogeneous metrics are not symmetric.

Proof. First, it is clear that SU(4) acts transitively on $S^{7}$, with isotropy group SU(3). Second, SU(4) acts transitively on $S^{5}$ via the double covering $\mathrm{SU}(4) \rightarrow \mathrm{SO}(6)$. The isotropy group of this action is the preimage of $\mathrm{SO}(5)$ under the covering, which can be identified with $\operatorname{Sp}(2)$. Now take the product action on $S^{5} \times S^{7}$. This is still transitive, for example because the restriction of the action of SU(4) on $S^{5}$ to $\mathrm{SU}(3)$ is still transitive. It follows that $S^{5} \times S^{7}$ is a homogeneous space of SU(4) with isotropy group $\mathrm{Sp}(2) \cap \mathrm{SU}(3)=\mathrm{SU}(2)$.

Proposition 5 implies that all SU(4)-homogeneous metrics on $S^{5} \times S^{7}$ are formal. By Remark 6, it follows that we cannot get the normal homogeneous metrics as product metrics. In particular, the metric on $S^{5} \times S^{7}$ that is the product of the symmetric space metrics on the factors, though also formal, is not normal homogeneous for the SU(4)-action.

To end this section, we want to show that Proposition 5 is sharp in the sense that it does not extend to arbitrary homogeneous spaces with a cohomology algebra of the form $\bigwedge(x, y)$ with $x$ of even degree and $y$ of odd degree.

A convenient class of examples to consider for this purpose are the so-called Aloff-Wallach spaces. These are homogeneous spaces of the form

$$
N_{k, l}=\mathrm{SU}(3) / T^{1},
$$

where $T^{1}$ is embedded as the diagonal matrices $D\left(z^{k}, z^{l}, z^{-k-l}\right)$, with $k$ and $l$ coprime integers with $k l(k+l) \neq 0$. Obviously, they are all homogeneous spaces of Cartan type, and are therefore [Kotschick and Terzić 2003] formal in the sense of Sullivan. It is also easy to see that they all have the real cohomology of $S^{2} \times S^{5}$. The name derives from [Aloff and Wallach 1975], where it proved that these spaces have homogeneous metrics of positive sectional curvature. The numerical conditions on $k$ and $l$ are there to make sure that $T^{1}$ acts on $\mathbb{C}^{3}$ without nonzero fixed points.

Since SU(3) endowed with a biinvariant metric is geometrically formal, it is natural to consider the submersion metrics on $\mathrm{SU}(3) / T^{1}$ which we get from the 
principal circle fibration

$$
T^{1} \rightarrow \mathrm{SU}(3) \stackrel{\pi}{\rightarrow} N_{k, l} .
$$

Such a metric is often called a normal homogeneous metric.

Theorem 8. The normal homogeneous metrics on Aloff-Wallach spaces are not formal.

Proof. Assume that $M=\mathrm{SU}(3) / T^{1}$ is geometrically formal for some embedding $T^{1} \subset \mathrm{SU}(3)$. Since $M$ has the real cohomology of $S^{2} \times S^{5}$, it carries two harmonic forms $\omega_{2}$ and $\omega_{5}$ such that $\omega_{2}^{2}=0$ and $\omega_{2} \wedge \omega_{5}$ is a volume form. Since $\mathrm{SU}(3)$ is simply connected, the Euler class $e$ of the principal bundle (1) is not zero, so that $e=\lambda\left[\omega_{2}\right]$ with $\lambda \neq 0$. We now normalize our form so that $\lambda=1$. In the following calculation we also ignore nonzero constants.

There exists a connection form $\alpha$ on the principal bundle (1) such that

$$
\pi^{*}\left(\omega_{2}\right)=d \alpha
$$

where $\pi$ is the projection in (1).

Let $\eta_{5}=\pi^{*}\left(\omega_{5}\right)$ and $\eta_{2}=\pi^{*}\left(\omega_{2}\right)$. Then $\eta_{2}$ and $\eta_{5}$ are closed and

$$
\eta_{2}^{2}=0
$$

Also,

$$
* \eta_{5}=* \pi^{*}\left(\omega_{5}\right)=\alpha \wedge \pi^{*}\left(* \omega_{5}\right)=\alpha \wedge \eta_{2}
$$

Then (2) gives

$$
d\left(* \eta_{5}\right)=d \alpha \wedge \eta_{2}=\eta_{2}^{2}=0 .
$$

This implies that $\eta_{3}=* \eta_{5}$ is a harmonic form on $\mathrm{SU}(3)$ with the biinvariant metric. Since the harmonic forms on SU(3) coincide with the biinvariant ones, we get that $\eta_{3}$ has the form

$$
\eta_{3}(X, Y, Z)=\langle X,[Y, Z]\rangle \quad \text { for } X, Y, Z \in \mathfrak{s u}(3) .
$$

On the other hand, we have a natural direct sum decomposition

$$
\mathfrak{s u}(3)=\mathfrak{t}^{1} \oplus\left(\mathfrak{s u}(3) / \mathfrak{t}^{1}\right),
$$

and (3) implies that there exists a 5-dimensional subspace $\mathscr{K}$ in $\mathfrak{s u}(3) / \mathfrak{t}^{1}$ such that $i_{v}\left(\eta_{3}\right)=0$ for any vector $v \in \mathscr{K}$.

Let $H_{1}, H_{2}, E_{1}, E_{2}, F_{1}, F_{2}$ be canonical (Chevalley) generators of the Lie algebra $\mathfrak{s u}(3)$. Consider the subspace $\mathscr{L}$ spanned by the vectors $H_{1}, H_{2}, E_{1}, F_{1}$. We are going to show that $i_{X}\left(\eta_{3}\right) \neq 0$ for any $X \in \mathscr{L}$. Any $X \in \mathscr{L}$ can be written in the 
form $X=a H_{1}+b H_{2}+c E_{1}+d F_{1}$. To prove our claim, we consider the following cases:

Case 1: If $d \neq 0$, then using the well-known relation between canonical generators of a simple Lie algebra and the root spaces related to the Killing form, we get $\eta_{3}\left(E_{1}, H_{1}, X\right)=-2 d\left\langle E_{1}, F_{1}\right\rangle \neq 0$.

Case 2: If $d=0$ and $c \neq 0$, then we have $\eta_{3}\left(F_{1}, H_{1}, X\right)=2 c\left\langle F_{1}, E_{1}\right\rangle \neq 0$.

Case 3: If $c=d=0$, then we have

$$
\eta_{3}\left(F_{1}, X, E_{1}\right)=(2 a-b)\left\langle F_{1}, E_{1}\right\rangle \quad \text { and } \quad \eta_{3}\left(F_{2}, X, E_{2}\right)=(2 b-a)\left\langle F_{2}, E_{2}\right\rangle \text {, }
$$

so there are always $E_{i}$ and $F_{i}$ for which $\eta_{3}\left(F_{i}, X, E_{i}\right) \neq 0$.

This implies that $\mathscr{L} \cap \mathscr{K}=0$, which is impossible for dimension reasons.

Remark 9. There are exactly two fibrations with fiber $S^{2}$ over $S^{5}$; cf. Section 5 . The trivial bundle $S^{2} \times S^{5}$ is of course geometrically formal with respect to product metrics. The nontrivial bundle is the 3 -symmetric space $\mathrm{SU}(3) / T^{1}$, where $T^{1}$ is embedded inside an $\mathrm{SU}(2) \subset \mathrm{SU}(3)$. In the above notation, this is the case $k=-l$, which is excluded in the definition of Aloff-Wallach spaces. Nevertheless, the argument above applies to show that a normal homogeneous metric is not geometrically formal.

Remark 10. The Aloff-Wallach spaces have interesting homogeneous metrics that are not normal homogeneous. These include metrics of positive sectional curvature [Aloff and Wallach 1975] and Einstein metrics, some of which admit Killing spinors. The latter metrics are not geometrically formal because of the following result, communicated to us by U. Semmelmann: A metrically formal Riemannian spin manifold $M$ of dimension $\geq 5$ admitting a nontrivial Killing spinor must have vanishing second Betti number.

It is known that a metric admitting a nontrivial Killing spinor must be Einstein, and thus is very special.

\section{Some algebraic topology of Stiefel manifolds}

In this section we prove that the homogeneous spaces listed in Theorem 2 are not homotopy equivalent to symmetric spaces or to products of real homology spheres, except for the second property in the case of $V_{3}\left(\mathbb{R}^{2 n}\right)$ with $n$ even. Together with Proposition 5 proved in the previous section, this completes the proof of Theorem 2.

Let us consider first the complex Stiefel manifolds $V_{2}\left(\mathbb{C}^{n}\right)=\mathrm{SU}(n) / \mathrm{SU}(n-2)$ consisting of orthonormal pairs of vectors for the standard Hermitian inner product on $\mathbb{C}^{n}$, with $n \geq 3$. Projecting such a pair to its first entry, we obtain a smooth 
fibration of $V_{2}\left(\mathbb{C}^{n}\right)$ over $S^{2 n-1}$ with fiber $S^{2 n-3}$. It is a classical problem in homotopy theory to determine when the total space of such a fiber bundle is homotopy equivalent to the product of base and fiber. In the case at hand, for even $n$ the action of the quaternions on $\mathbb{C}^{n}=\mathbb{H}^{n / 2}$ defines a section of the fibration, which splits the long exact homotopy sequence and makes $V_{2}\left(\mathbb{C}^{n}\right)$ indistinguishable from $S^{2 n-1} \times S^{2 n-3}$ at the level of homotopy groups. However, the following result was proved by James and Whitehead [1954] modulo Adams's solution of the Hopf invariant-one problem:

Theorem 11 [James and Whitehead 1954; Adams 1960]. For $n \geq 3$, the Stiefel manifold $V_{2}\left(\mathbb{C}^{n}\right)=\mathrm{SU}(n) / \mathrm{SU}(n-2)$ is not homotopy equivalent to $S^{2 n-1} \times S^{2 n-3}$, unless possibly if $n=4$.

In fact, James and Whitehead proved that if $V_{2}\left(\mathbb{C}^{n}\right)=\mathrm{SU}(n) / \mathrm{SU}(n-2)$ is homotopy equivalent to $S^{2 n-1} \times S^{2 n-3}$, then $\pi_{4 n-1}\left(S^{2 n}\right)$ contains an element of Hopf invariant one. By the result of Adams, it follows that $n$ is in $\{1,2,4\}$. This combination of results is also mentioned in [James 1971, Theorem 1.7], but the exceptional case is misstated there. In the notation of [James 1971], the exceptional case should be denoted $n=4$ and $k=2$, not $n=k=2$.

Remark 12. Some years after the results of [James and Whitehead 1954] and [Adams 1960], Gilmore [1967] showed that $\pi_{2 n-2}\left(V_{2}\left(\mathbb{C}^{n}\right)\right)$ is trivial for odd $n$. Since $\pi_{2 n-2}\left(S^{2 n-1} \times S^{2 n-3}\right)=\pi_{2 n-2}\left(S^{2 n-3}\right)=\mathbb{Z}_{2}$, this gives another proof of Theorem 11 for odd $n$. As we remarked earlier, there is no such proof in the case of even $n$.

The arguments of James and Whitehead only show that the existence of an element of Hopf invariant one is necessary for $V_{2}\left(\mathbb{C}^{n}\right)=\mathrm{SU}(n) / \mathrm{SU}(n-2)$ to be homotopy equivalent to $S^{2 n-1} \times S^{2 n-3}$. It turns out that this condition is in fact sufficient not just for homotopy equivalence, but for diffeomorphism. This following result completes the proof of case (3) in Theorem 2.

Theorem 13. If a complex Stiefel manifold $V_{2}\left(\mathbb{C}^{n}\right)=\mathrm{SU}(n) / \mathrm{SU}(n-2)$ with $n \geq 3$ is homotopy equivalent to a symmetric space or to a product of real homology spheres, then $n=3$ or $n=4$. In the first case, $V_{2}\left(\mathbb{C}^{3}\right)=\mathrm{SU}(3)$ is a symmetric space not homotopy equivalent to a product of real homology spheres. In the second case, $V_{2}\left(\mathbb{C}^{4}\right)=\mathrm{SU}(4) / \mathrm{SU}(2)$ is diffeomorphic to $S^{5} \times S^{7}$.

Proof. Suppose that $V_{2}\left(\mathbb{C}^{n}\right)$ is homotopy equivalent to a product $X_{1} \times X_{2}$ of real homology spheres. Then because $V_{2}\left(\mathbb{C}^{n}\right)$ is simply connected, so are both the $X_{i}$. Moreover, because $V_{2}\left(\mathbb{C}^{n}\right)$ has the integral homology of a product of spheres, it follows that each $X_{i}$ is an integral homology sphere. Thus the $X_{i}$ are homotopy spheres. 
Now, if $V_{2}\left(\mathbb{C}^{n}\right)$ is homotopy equivalent to a product of (homotopy) spheres, then Theorem 11 gives $n=4$. Conversely, $V_{2}\left(\mathbb{C}^{4}\right)$ is an $S^{5}$ bundle over $S^{7}$ with structure group SU(4). The corresponding clutching map gives an element of $\pi_{6}(\mathrm{SU}(4))$, which is trivial [Borel and Serre 1953]. Thus $V_{2}\left(\mathbb{C}^{4}\right)$ is diffeomorphic to the total space of the trivial bundle $S^{5} \times S^{7}$; compare with Proposition 7 .

Next suppose that $V_{2}\left(\mathbb{C}^{n}\right)$ is homotopy equivalent to a symmetric space. Onishchik [1963] and Kramer [2002] have classified the homogeneous spaces with the cohomology of a product of odd-dimensional spheres. If we assume that $G / H$ has the integral cohomology of such a product, then apart from products of spheres and a handful of symmetric spaces of dimension $\leq 14$, the remaining examples are exactly the spaces listed as (3)-(6) in Theorem 2 and the symmetric space $E_{6} / F_{4}$ with the cohomology of $S^{9} \times S^{17}$. If $n \geq 5$, then the dimension of $V_{2}\left(\mathbb{C}^{n}\right)$ is $\geq 16$, so we do not have to consider the sporadic irreducible symmetric spaces of dimension $\leq 14$ arising in the classification of Onishchik and Kramer. Similarly, $V_{2}\left(\mathbb{C}^{n}\right)$ cannot be homotopy equivalent to $E_{6} / F_{4}$ for dimension reasons. If $V_{2}\left(\mathbb{C}^{n}\right)$ were homotopy equivalent to a reducible symmetric space, then each factor would be a homotopy sphere, and we would be in the case excluded already.

Finally, $V_{2}\left(\mathbb{C}^{3}\right)=\mathrm{SU}(3)$ is of course a symmetric space, but it is not homotopy equivalent to a product of spheres. For if it were, then the spheres would have dimensions 3 and 5, leading to a contradiction with $\pi_{6}(\mathrm{SU}(3))=\mathbb{Z}_{6}$ (cf. [Borel and Serre 1953]), since $\pi_{6}\left(S^{3}\right)=\mathbb{Z}_{12}$.

In a similar way one proves the corresponding result for the quaternionic Stiefel manifolds. The statement is cleaner since an exceptional case not excluded by the Hopf invariant does in fact not occur. This following result completes the proof of case (4) in Theorem 2.

Theorem 14. No quaternionic Stiefel manifold $V_{2}\left(\mathbb{M}^{n}\right)=\operatorname{Sp}(n) / \operatorname{Sp}(n-2)$ with $n \geq 2$ is homotopy equivalent to a product of real homology spheres. If it is homotopy equivalent to a symmetric space, then $n=2$.

Proof. If $V_{2}\left(\mathbb{M}^{n}\right)$ is homotopy equivalent to a nontrivial product, then as in the previous proof, we may assume that each factor is a sphere. Assuming that $V_{2}\left(\mathbb{M}^{n}\right)$ is homotopy equivalent to $S^{4 n-5} \times S^{4 n-1}$, James and Whitehead proved in [1954, Theorem 1.21] that $\pi_{8 n-1}\left(S^{4 n}\right)$ contains an element of Hopf invariant one. The result of Adams [1960] then implies that $n=2$. However, for $n=2$, we have $V_{2}\left(\mathbb{H}^{2}\right)=\operatorname{Sp}(2)$, and this is not homotopy equivalent to a product of spheres, for example because $\pi_{6}(\mathrm{Sp}(2))$ is trivial as first proved by Borel and Serre [1953].

Next suppose that $V_{2}\left(\mathbb{M}^{n}\right)$ is homotopy equivalent to a symmetric space. If $n \geq 3$, then the dimension of $V_{2}\left(\mathbb{H}^{n}\right)$ is $\geq 18$, so that, again, we do not have to consider the sporadic irreducible symmetric spaces of dimension $\leq 14$ arising in the classification due to Onishchik [1963] and Kramer [2002]. For dimension reasons, $E_{6} / F_{4}$ 
cannot occur either. In the reducible case $V_{2}\left(\mathbb{Q}^{n}\right)$ would be homotopy equivalent to a product of spheres, and this we have excluded already. Finally, for $n=2$ we have the symmetric space $V_{2}\left(\mathbb{H}^{2}\right)=\operatorname{Sp}(2)$.

Remark 15. Instead of using the results of James-Whitehead [1954] and Adams [1960], we could, in most cases, appeal to the calculations of Oguchi [1969]. On the one hand we have $\pi_{4 n-2}\left(S^{4 n-1} \times S^{4 n-5}\right)=\pi_{4 n-2}\left(S^{4 n-5}\right)=\mathbb{Z}_{24}$ for $n \geq 3$. On the other, by [Ōguchi 1969], $\pi_{4 n-2}\left(V_{2}\left(\mathbb{N}^{n}\right)\right)=\mathbb{Z}_{d}$, where $d=\operatorname{gcd}\{n, 24\}$. Thus, whenever $n$ is not divisible by 24 , one concludes that $V_{2}\left(\mathbb{D}^{n}\right)$ is not homotopy equivalent to a product of spheres.

To complete the proof of case (5) in Theorem 2, we prove the following:

Theorem 16. The octonionic Stiefel manifold $V_{2}\left(\mathbb{O}^{2}\right)=\operatorname{Spin}(9) / G_{2}$ is not homotopy equivalent to a symmetric space, or to a product of real homology spheres.

Proof. This manifold has the integral homology of $S^{7} \times S^{15}$. As in the previous proofs, if $V_{2}\left(\mathbb{O}^{2}\right)$ were homotopy equivalent to a nontrivial product, then this product would have to be $S^{7} \times S^{15}$. We now prove that $V_{2}\left(\mathbb{O}^{2}\right)$ and $S^{7} \times S^{15}$ are in fact not homotopy equivalent, distinguished by their homotopy groups.

On the one hand, we have

$$
\pi_{14}\left(S^{7} \times S^{15}\right)=\pi_{14}\left(S^{7}\right)=\mathbb{Z}_{120} .
$$

On the other hand, we will show that $\pi_{14}\left(V_{2}\left(\mathbb{O}^{2}\right)\right)$ has order at most 16 . For this we consider the principal $G_{2}$ bundle with total space $\operatorname{Spin}(9)$ over $V_{2}\left(\mathbb{O}^{2}\right)$, and the following piece of its exact homotopy sequence:

$$
\cdots \rightarrow \pi_{14}(\operatorname{Spin}(9)) \rightarrow \pi_{14}\left(V_{2}\left(\mathbb{O}^{2}\right)\right) \rightarrow \pi_{13}\left(G_{2}\right) \rightarrow \cdots
$$

The group on the right is trivial and the group on the left is $\mathbb{Z}_{8} \oplus \mathbb{Z}_{2}$, by calculations of [Mimura 1967].

The proof that $V_{2}\left(\mathbb{O}^{2}\right)$ is not homotopy equivalent to a symmetric space is the same as for the complex and quaternionic Stiefel manifolds. First of all, reducible symmetric spaces cannot arise because $V_{2}\left(\mathbb{O}^{2}\right)$ is not homotopy equivalent to a product, as we just proved. Second of all, there is no irreducible symmetric space with the correct cohomology.

Next we deal with the space $\operatorname{Spin}(10) / \operatorname{Spin}(7)$, case (6) in Theorem 2.

Theorem 17. The quotient $\operatorname{Spin}(10)$ / Spin(7) is not homotopy equivalent to a symmetric space, or to a product of real homology spheres.

Proof. Consider the principal Spin(9) bundle $\operatorname{Spin}(10) \rightarrow \operatorname{Spin}(10) / \operatorname{Spin}(9)=S^{9}$. The spin representation of $\operatorname{Spin}(9)$ on $\mathbb{R}^{16}$ associates to this principal bundle a real vector bundle $V$ of rank 16 . Our homogeneous space $X=\operatorname{Spin}(10) / \operatorname{Spin}(7)$ is the unit sphere bundle in $V$, with fiber $\operatorname{Spin}(9) / \operatorname{Spin}(7)=S^{15}$. 
The manifold $X$ has the integral homology of $S^{9} \times S^{15}$. As in the previous proofs, if $X$ were homotopy equivalent to a nontrivial product, then this product would have to be $Y=S^{9} \times S^{15}$. We will show below that in fact $X$ and $Y$ are not homotopy equivalent. The proof that $X$ is not homotopy equivalent to a symmetric space is then the same as for the complex and quaternionic Stiefel manifolds. First, reducible symmetric spaces cannot arise because $X$ is not homotopy equivalent to a product. Second, there is no irreducible symmetric space with the correct cohomology.

Now we begin the proof that $X$ and $Y=S^{9} \times S^{15}$ are not homotopy equivalent. The principal bundle $\operatorname{Spin}(10) \rightarrow S^{9}$ corresponds to an element of $\pi_{8}(\operatorname{Spin}(9))$, whose image in $\pi_{8}(\mathrm{SO}(16))$ classifies $V$. Since $V$ is nontrivial, this element is the nontrivial element of $\pi_{8}(\mathrm{SO}(16))=\mathbb{Z}_{2}$.

Recall that the fibration $X \longrightarrow S^{9}$ has a section for dimension reasons. Now [James and Whitehead 1954, Corollary 1.9] tells us that $X$ is homotopy equivalent to $Y=S^{9} \times S^{15}$ if and only if a certain invariant $\lambda(X)$ vanishes. This invariant is an element in the group $\Lambda_{9,15}=\operatorname{Im} J / \operatorname{Im} P$, where $J: \pi_{8}(\operatorname{SO}(15)) \rightarrow \pi_{23}\left(S^{15}\right)$ is the classical $J$-homomorphism and $P: \pi_{9}\left(S^{15}\right) \rightarrow \pi_{23}\left(S^{15}\right)$ is, in our case, the zero map, as its domain is zero. Thus, in our case, $\lambda(X) \in J\left(\pi_{8}(\operatorname{SO}(15))\right.$, and we only have to determine whether this is zero. Unravelling the definition of $\lambda$ given in [James and Whitehead 1954], we find the following: if we identify $\pi_{8}(\operatorname{SO}(15))$ with $\pi_{8}(\mathrm{SO}(16))$, then $\lambda(X)$ is just the image under the classical $J$-homomorphism of the classifying element of $V$ in $\pi_{8}(\mathrm{SO}(16))$. Now $\pi_{8}(\mathrm{SO}(15))$ has order 2 , and we know that the image of the $J$-homomorphism in this degree is the group $J\left(S^{9}\right)$, also of order 2, cf. [Bott 1969; Husemoller 1975]. Thus the $J$-homomorphism is an isomorphism, and $\lambda(X) \neq 0$ because the classifying map of $V$ represents the nonzero element of $\pi_{8}(\mathrm{SO}(16))$.

Remark 18. The existence of a section to the fibration $S^{15} \rightarrow X \stackrel{\pi}{\rightarrow} S^{9}$ implies that $X$ and $Y=S^{9} \times S^{15}$ have isomorphic homotopy groups. Thus there can be no easy argument to prove that they are not homotopy equivalent.

It remains to discuss the homotopy types of the real Stiefel manifolds occurring in cases (1) and (2) of Theorem 2. They are geometrically formal by Proposition 5, and they are not homotopy equivalent to products of spheres because of the presence of torsion in their integral homology. However, for these manifolds it is more difficult to exclude the homotopy equivalence to products of real homology spheres, because the factors in such a decomposition would not necessarily be homotopy spheres. The following result completes the proof of Theorem 2 .

Theorem 19. The real Stiefel manifolds $V_{4}\left(\mathbb{R}^{2 n+1}\right)$ and $V_{3}\left(\mathbb{R}^{2 n}\right)$ with $n \geq 3$ are not homotopy equivalent to symmetric spaces. They are not homotopy equivalent to nontrivial products, except possibly for $V_{3}\left(\mathbb{R}^{2 n}\right)$ with $n$ even. 
Proof. These manifolds have the real cohomology algebras of $S^{4 n-5} \times S^{4 n-1}$ and $S^{2 n-1} \times S^{4 n-5}$, respectively. Therefore, if one of them is homotopy equivalent to a nontrivial product, then the factors are real homology spheres of dimensions $4 n-5$ and $4 n-1$, respectively $2 n-1$ and $4 n-5$.

For the real Stiefel manifolds the cohomology with coefficients in $\mathbb{Z}_{2}$ and the Steenrod operations on it were determined by Borel [1953a]. From the structure of the Steenrod operations, Hsiang and Su [1968] deduced that $V_{4}\left(\mathbb{R}^{2 n+1}\right)$ and $V_{3}\left(\mathbb{R}^{2 n}\right)$ cannot be homotopy equivalent to nontrivial products, except possibly in the second case, when $n$ is even.

Suppose that one of these manifolds is homotopy equivalent to a symmetric space. Then this symmetric space is reducible, and we are in the exceptional case where $V_{3}\left(\mathbb{R}^{2 n}\right)$ could be homotopy equivalent to a product. To see this, consider the irreducible symmetric spaces. Computations of their real cohomology algebras [Borel 1953b; Takeuchi 1962] or of their rational homotopy groups [Terzić 2003] show that, among them, only

$$
\mathrm{SU}(5) / \mathrm{SO}(5), \quad \mathrm{SU}(6) / \mathrm{Sp}(3), \quad E_{6} / F_{4}, \quad \mathrm{Sp}(2), \quad \mathrm{SU}(3), \quad G_{2}, \quad \operatorname{Spin}(4)
$$

have the real cohomology algebra of a product of odd-dimensional spheres, with the dimensions given by the pairs
$(5,9)$,
$(5,9)$
$(9,17)$,
$(3,7)$,
$(3,5)$,
$(3,11)$,
$(3,3)$,

respectively. None of these dimension pairs is of the form $(4 n-5,4 n-1)$ or $(2 n-1,4 n-5)$ with $n \geq 3$. Therefore, $V_{4}\left(\mathbb{R}^{2 n+1}\right)$ or $V_{3}\left(\mathbb{R}^{2 n}\right)$ cannot be homotopy equivalent to any irreducible compact simply connected symmetric space.

Finally we have to consider the possibility that $V_{3}\left(\mathbb{R}^{2 n}\right)$ with $n$ even could be homotopy equivalent to a reducible symmetric space. Then each factor would be a compact symmetric space with the real cohomology of $S^{2 n-1}$, respectively $S^{4 n-5}$. Since $V_{3}\left(\mathbb{R}^{2 n}\right)$ has torsion in its integral homology, at least one of the factors has to be a simply connected symmetric space having the real cohomology of a sphere, but different integral cohomology. According to the classification, due to Onishchik [1963; 1995], of homogeneous spaces whose real cohomology is that of a sphere, the only possibility is $\mathrm{SU}(3) / \mathrm{SO}(3)$, of dimension 5. For even $n$, neither $2 n-1$ nor $4 n-1$ can equal 5 , and this contradiction completes the proof.

Remark 20. Projection of a 3-frame onto its first entry defines a smooth fibration of $V_{3}\left(\mathbb{R}^{2 n}\right)$ over $S^{2 n-1}$, with fiber $V_{2}\left(\mathbb{R}^{2 n-1}\right)$. Note that the fiber $V_{2}\left(\mathbb{R}^{2 n-1}\right)$ is a real homology sphere, but not an integral one. As far as we know, it is still an open problem whether for some even $n$ the total space could be homotopy equivalent, or even diffeomorphic, to the product of base and fiber; see the discussion in 
[James 1971]. If this happens for some $n$, then by a result of James [1971], this $n$ is not just even, but a power of 2 .

Onishchik [1963] and Kramer [2002] have classified all the compact homogeneous spaces with the real cohomology of a product of odd-dimensional spheres. In addition to nontrivial products and the spaces we have discussed already in Theorem 2 and its proof, there is a large number of sporadic cases that have the real cohomology of a product of spheres but different integral cohomology. All these spaces are geometrically formal by Proposition 5. To end this section, we discuss the diffeomorphism type of one of these sporadic examples.

Consider the composition of standard inclusions $\mathrm{SO}(3) \subset \mathrm{SU}(3) \subset \mathrm{SU}(4)$. The quotient $X=\mathrm{SU}(4) / \mathrm{SO}(3)$ has the real cohomology of $S^{5} \times S^{7}$, but different integral cohomology.

Proposition 21. The manifold $X=\mathrm{SU}(4) / \mathrm{SO}(3)$ is not diffeomorphic to a nontrivial product, nor to a symmetric space.

Proof. Suppose $X$ were diffeomorphic to some nontrivial product. Then the factors would have to be simply connected real homology spheres of dimensions 5 and 7 , respectively. Since we are assuming that $X$ is diffeomorphic to the product, not just homotopy equivalent to it, the factors must in fact be homogeneous spaces. Therefore, all the candidates must occur in the classification of Onishchik and Kramer. In dimension 5, the candidates are $S^{5}$ and $\mathrm{SU}(3) / \mathrm{SO}(3)$; in dimension 7, they are $S^{7}, V_{2}\left(\mathbb{R}^{5}\right)$ and $\mathrm{Sp}(2) / \mathrm{Sp}(1)$ with a nonstandard embedding of the subgroup. Looking at the third homotopy groups, we have $\pi_{3}(X)=\pi_{3}(\mathrm{SU}(3) / \mathrm{SO}(3))=\mathbb{Z}_{4}$, but $\pi_{3}\left(V_{2}\left(\mathbb{R}^{5}\right)\right)=\mathbb{Z}_{2}$ and $\pi_{3}(\operatorname{Sp}(2) / \operatorname{Sp}(1))=\mathbb{Z}_{10}$; cf. [Kramer 2002, page 65]. Thus, on the one hand, the only product of homogeneous spaces that has the same third homotopy group as $X$ is $S^{7} \times \mathrm{SU}(3) / \mathrm{SO}(3)$. On the other hand, $X$ and $S^{7} \times \mathrm{SU}(3) / \mathrm{SO}(3)$ have different sixth homotopy groups. This is so because the exact homotopy sequence of the fibration $\mathrm{SU}(4) \rightarrow X$ shows that $\pi_{6}(X)=\pi_{5}(\mathrm{SO}(3))$, which is of order 2 , whereas $\pi_{6}(\mathrm{SU}(3) / \mathrm{SO}(3))$ is known to be of order 4; see [Lundell 1992].

Finally, if $X$ were diffeomorphic to a symmetric space, then by what we just proved, that symmetric space would have to be irreducible. However, by the classification of irreducible symmetric spaces, there is no such space with the real cohomology of $S^{5} \times S^{7}$.

Note that if we discuss only the homotopy type of $X=\mathrm{SU}(4) / \mathrm{SO}(3)$, then we have to consider products of real homology spheres that are not necessarily homogeneous, and the argument breaks down. 


\section{Biquotients}

Let $G$ be a compact Lie group, and $U$ a closed subgroup of $G \times G$ that acts freely on $G$ by $\left(u_{1}, u_{2}\right) \cdot g=u_{1} g u_{2}^{-1}$. Then the orbit space is a smooth manifold denoted by $G / U$ and is called a biquotient of $G$. A biinvariant metric on $G$ descends to a metric on any biquotient. More generally, one can consider subgroups $U \subset \operatorname{ISO}(G)$ acting freely. In some cases $\operatorname{ISO}(G)$ is strictly larger than $G \times G$, so one gets more examples.

Biquotients were first studied systematically by Eschenburg [1992a; 1992b] as a source of examples of manifolds with positive sectional curvature. Among Eschenburg's biquotients in [Eschenburg 1992a] there are several examples for which we can prove easily that they are not geometrically formal.

Example 22. There is a 6-dimensional example $M=G / U$ whose real cohomology is very similar but not isomorphic to that of $S U(3) / T^{2}$, considered in [Kotschick and Terzić 2003]. Moreover, $M$ and SU(3) $/ T^{2}$ have the same integral homology groups and the same cohomology algebra mod 2. We can show that this biquotient is not geometrically formal by using almost the same argument as the one we used for $\mathrm{SU}(3) / T^{2}$ in [Kotschick and Terzić 2003].

Let $G=U(3)$ and

$$
U \subset\left\{(D(a, a, \bar{a}), D(b, c, 1)) ; a, b, c \in S^{1}\right\} \subset G \times G .
$$

Eschenburg [1992a] proved that $H^{*}(G / U)$ is generated by two generators $x$ and $y$ of degree 2 with the relations $x y=y^{2}-x^{2}$ and $x^{3}=0$. Setting $z=(1 / \sqrt{5})(x-2 y)$, we get $z^{2}=x^{2}$ and $z^{3}=-(2 / \sqrt{5}) x y^{2} \neq 0$. If $G / U$ is geometrically formal, then it has two 2-forms $x$ and $z$, such that $x^{3}=0, x^{2}=z^{2}$, and $z^{3}$ is a volume form.

If $G / U$ were geometrically formal, then for a formal metric these relations would hold pointwise for the harmonic forms representing the cohomology classes $x$ and $z$ and their products. The form $x$ would then have rank 4 everywhere. If $v$ were a vector in its kernel, the relation $x^{2}=z^{2}$ would show that $i_{v} z \wedge z=0$, which would contradict the fact that $z^{3}$ would have to be a volume form.

The second example uses a different argument to obstruct geometric formality, related to symplectic structures defined by harmonic forms of formal metrics.

Example 23. Let $G=\mathrm{SU}(3)$ and

$$
U=T^{1} \times T^{1}=\left\{D\left(a^{k}, a^{l}, a^{-k-l}\right), D\left(b^{m}, b^{n}, b^{-m-n}\right) ; a, b \in S^{1}\right\} .
$$

Using the results of [Eschenburg 1992a] on the cohomology of biquotients, it is easy to compute $H^{*}(G / U)$. We get the following algebra structure: There are two linearly independent generators $x$ and $y$ in degree 2, subject to the relations $x^{2}=y^{2}$ and $x^{3}=y^{3}$. Then $H^{4}$ is spanned by $x y$ and $x^{2}=y^{2}$, and $H^{6}$ is spanned 
by $x^{3}=y^{3}=x^{2} y=x y^{2}$. If we assume that $M$ is geometrically formal, then the harmonic form representing $x+y$ is a symplectic form. It then follows from $(x-y)(x+y)=0$ that $x-y$ vanishes, which contradicts the linear independence of $x$ and $y$, because on a symplectic six-manifold the wedge product with the symplectic form is an isomorphism between two-forms and four-forms. Thus $M$ cannot be geometrically formal.

Next we consider Totaro's biquotients of $S^{3} \times S^{3} \times S^{3}$, which he studied in [2003] as an example of a family of 6-manifolds with nonnegative sectional curvature, but with infinitely many distinct isomorphism classes of rational cohomology rings. For all these manifolds we prove that they cannot be geometrically formal because of the structure of their cohomology rings.

Theorem 24. The biquotients studied in [Totaro 2003] are not geometrically formal.

Proof. The rational cohomology ring of the biquotients that Totaro considers has three generators $x_{1}, x_{2}$ and $x_{3}$ in degree 2, satisfying the relations

$$
\begin{aligned}
x_{1}^{2} & =0, \\
a x_{1} x_{2}+x_{2} x_{3}+x_{2}^{2} & =0, \\
b x_{1} x_{3}+2 x_{2} x_{3}+x_{3}^{2} & =0,
\end{aligned}
$$

for some integers $a$ and $b$; see [Totaro 2003]. If we assume that these manifolds are geometrically formal, then the same relations (7) hold at the level of their representative harmonic forms, which we denote by the same letters. In order to prove that geometric formality leads to a contradiction, we differentiate the following cases according to the values of the constants $a$ and $b$.

Case 1: We first consider the case when $a \cdot b \neq 0$. Then we can obviously normalize $x_{1}$ so that $a$ becomes 1 . Consider the forms

$$
y_{1}=x_{1}+\frac{3}{b} x_{2} \quad \text { and } \quad y_{2}=x_{1}+\frac{3}{2} x_{3} .
$$

Using the relations (7), we see that $y_{1}^{3}=y_{2}^{3}=0$, while $x_{1} y_{1}^{2}, x_{1} y_{2}^{2}$ and $y_{1} y_{2}^{2}$ are volume forms.

Since the cubes of $y_{1}$ and $y_{2}$ vanish, we have $\operatorname{dim} \operatorname{Ker}\left(y_{1}\right)=\operatorname{dim} \operatorname{Ker}\left(y_{2}\right)=2$ and, since $y_{1} y_{2}^{2}$ is a volume form on $M$, it follows that $\operatorname{Ker}\left(y_{1}\right) \cap \operatorname{Ker}\left(y_{2}\right)=0$.

If we rewrite the relations (7) in terms of $x_{1}, y_{1}$ and $y_{2}$, we find, after some straightforward calculations, that

$$
\begin{aligned}
x_{1}^{2} & =0, \\
(1-2 b) x_{1} y_{1}-2 x_{1} y_{2}+2 y_{1} y_{2}+b y_{1}^{2} & =0, \\
-2 b x_{1} y_{1}+(b-4) x_{1} y_{2}+2 b y_{1} y_{2}+2 y_{2}^{2} & =0 .
\end{aligned}
$$


If we multiply the first relation by $b-4$, and add it to the second one multiplied by 2 , we get

$$
\left(5 b-2 b^{2}-4\right) x_{1} y_{1}+(6 b-8) y_{1} y_{2}+b(b-4) y_{1}^{2}+4 y_{2}^{2}=0 .
$$

Let $u_{1} \in \operatorname{Ker}\left(y_{1}\right)$ and $u_{2} \in \operatorname{Ker}\left(y_{2}\right)$ be such that $u_{2} \notin \mathbb{R}\left\{u_{1}\right\} \oplus \operatorname{Ker}\left(x_{1}\right)$ (such a $u_{2}$ always exists, otherwise we would have $\operatorname{Ker}\left(x_{1}\right) \cap \operatorname{Ker}\left(y_{2}\right) \neq 0$ which is a contradiction with the fact that $x_{1} y_{2}^{2}$ is a volume form).

In other words, $T_{p} M=\left\{u_{1}, u_{2}\right\} \oplus \operatorname{Ker}\left(x_{1}\right)$. Then if we contract the equation (8) with $u_{1}$ and $u_{2}$, we get

$$
\left(5 b-2 b^{2}-4\right) i_{u_{2}}\left(y_{1} \wedge i_{u_{1}} x_{1}\right)+(6 b-8) i_{u_{2}} y_{1} \wedge i_{u_{1}} y_{2}=0 .
$$

Look at the 1-forms $i_{u_{2}} y_{1}$ and $i_{u_{1}} y_{2}$. Since the dimension of the kernel of each of them is $\geq 5$, it follows that the intersection $L$ of their kernels has dimension $\geq 4$. Since $x_{1}^{2}=0$, it follows that $\operatorname{dim} \operatorname{Ker}\left(x_{1}\right)=4$, and thus $\operatorname{dim}\left(\operatorname{Ker}\left(x_{1}\right) \cap L\right) \geq 2$.

Let $w \in \operatorname{Ker}\left(x_{1}\right) \cap L$. If we contract (9) with $w$, we get $x_{1}\left(u_{1}, u_{2}\right) i_{w} y_{1}=0$, since the coefficient $5 b-2 b^{2}-4$ has no real zeros.

This is in contradiction with the fact that $x_{1} y_{1}^{2}$ is a volume form. Namely, take $w_{1}, w_{2}, w_{3} \in \operatorname{Ker}\left(x_{1}\right)$ such that together with $u_{1}, u_{2}, w$, they form a basis of $T_{p} M$. Then we have $x_{1} y_{1}^{2}\left(u_{1}, u_{2}, w, w_{1}, w_{2}, w_{3}\right)=0$, which is impossible.

Case 2: If $a=0$ and $b \neq 0$, we first normalize $x_{1}$ to get $b=1$ and proceed as in the previous case, taking $y_{1}=x_{1}+3 x_{2}$ and $y_{2}=x_{1}+6 x_{3}$.

Case 3: If $a \neq 0$ and $b=0$, we again first normalize $x_{1}$ to have $a=1$, and take $y_{1}=x_{2}$ and $y_{2}=x_{1}+\frac{3}{2} x_{3}$.

Case 4: For $a=b=0$, we have the following relations in cohomology:

$$
x_{1}^{2}=0, \quad x_{2} x_{3}+x_{2}^{2}=0, \quad 2 x_{2} x_{3}+x_{3}^{2}=0 .
$$

Take $y_{1}=x_{2}+x_{3}$ and $y_{2}=x_{2}+\frac{1}{2} x_{3}$. The cohomology relations imply that $y_{1}^{3}=y_{2}^{3}=0$, and that $y_{1} y_{2}^{2}$ is a volume form on $M$. If we rewrite these relations in terms of $y_{1}$ and $y_{2}$, we obtain

$$
y_{2}^{2}-y_{1} y_{2}=0 \quad \text { and } \quad y_{1}^{2}-2 y_{1} y_{2}=0 .
$$

Now take $u \in \operatorname{Ker}\left(y_{1}\right)$. The relations imply that $i_{u} y_{2}^{2}=0$, which contradicts that $y_{1} y_{2}^{2}$ is a volume form.

\section{Two-sphere bundles over the complex projective plane}

For our calculations in [Kotschick and Terzić 2003], the example SU(3)/ $T^{2}$ was the crucial case, from which all others were derived by various generalizations. The inclusions $T^{2} \hookrightarrow U(2) \hookrightarrow \mathrm{SU}(3)$ show that $\mathrm{SU}(3) / T^{2}$ fibers over $\mathbb{C} P^{2}$ with 
fiber $S^{2}$. In fact, this fibration is well known as the twistor fibration of $\mathbb{C} P^{2}$; compare with [Kotschick and Terzić 2009]. We now generalize to arbitrary 2-sphere fibrations over $\mathbb{C} P^{2}$.

Assume first that we have an arbitrary smooth oriented fibration with fiber $S^{2}$. Because the orientation-preserving diffeomorphism group of $S^{2}$ is homotopy equivalent to $\mathrm{SO}(3)$, we may assume that the structure group is $\mathrm{SO}(3)$. Then $M$ is the unit sphere bundle in the associated rank-3 vector bundle $V$.

As $\mathrm{SO}(3)$ coincides with the projective unitary group $\mathrm{PU}(2)$, every 2-sphere bundle is the projectivization of a complex rank-2 vector bundle $E$. One can recover $V$ by passing to the adjoint bundle. We have

$$
w_{2}(V)=c_{1}(E)(\bmod 2) \text { and } p_{1}(V)=c_{1}^{2}(E)-4 c_{2}(E) .
$$

In many cases, for example when $w_{2}(V)$ is nontrivial and $p_{1}(V)$ is divisible by 8 , it follows from [Grove and Ziller 2008, Theorem C] that $M$ admits a cohomogeneityone action of $\mathrm{SO}(3) \times \mathrm{SO}(3)$. Thus, these manifolds are highly symmetric, and are, in this sense, close to homogeneous.

Theorem 25. Let $M^{6}$ be the total space of an $S^{2}$ bundle $E$ over $\mathbb{C} P^{2}$. Then $M$ is geometrically formal if and only if it is the trivial bundle $S^{2} \times \mathbb{C} P^{2}$.

Proof. Assume $M^{6}$ is the total space of the projectivization of a complex rank-2 vector bundle $E$ over $\mathbb{C} P^{2}$. Then the cohomology of $M$ is multiplicatively generated by two degree 2 classes $x$ and $y$, where we use $x$ for the generator pulled back from $\mathbb{C} P^{2}$ and $y$ for a class that restricts as a generator to every fiber. By the definition of Chern classes we choose $y$ so that

$$
y^{2}+c_{1}(E) x y+c_{2}(E) x^{2}=0,
$$

where, by an obvious abuse of notation, we use $c_{i}(E)$ for the Chern numbers $\left\langle c_{i}(E),\left[\mathbb{C} P^{i}\right]\right\rangle$. We also have $x^{3}=0$.

After replacing $y$ by a linear combination of $x$ and $y$ we may assume that

$$
y^{2}+c x^{2}=0
$$

where $c=-\frac{1}{4} p_{1}(V)$ vanishes if and only if $M$ is the trivial bundle. To pass from (10) to (11), we can twist $E$ by a line bundle. This does not affect the projectivization, but we can kill the first Chern class if $w_{2}(V)=0$. If this is not the case, we can still make the required base change, which amounts to twisting by a virtual line bundle whose Chern class is half-integral. In this case the constant $c$ is not integral.

Now (11) together with $x^{3}=0$ implies that $x y^{2}=0$. We also have $y^{3}+c x^{2} y=0$. If $c \neq 0$, we conclude that $y^{3} \neq 0$, for otherwise there would be no degree 6 cohomology in $M$. Thus, if $c \neq 0$ and $M$ is geometrically formal, then the harmonic 
2-form representing $y$ is nondegenerate. However, since $x^{3}=0$ the harmonic form representing $x$ has a nontrivial kernel and, if we contract (11) with an element $v$ in this kernel, we deduce $2 i_{v} y \wedge y=0$, which contradicts the nondegeneracy of $y$.

Thus we have proved that a nontrivial $M$ cannot be geometrically formal. Conversely, the trivial bundle $S^{2} \times \mathbb{C} P^{2}$ is geometrically formal with respect to a product of Kähler metrics; cf. [Kotschick 2001]. In fact, it is a symmetric space.

\section{References}

[Adams 1960] J. F. Adams, "On the non-existence of elements of Hopf invariant one", Ann. of Math. (2) 72 (1960), 20-104. MR 25 \#4530 Zbl 0096.17404

[Aloff and Wallach 1975] S. Aloff and N. R. Wallach, "An infinite family of distinct 7-manifolds admitting positively curved Riemannian structures", Bull. Amer. Math. Soc. 81 (1975), 93-97. MR 51 \#6851 Zbl 0362.53033

[Besse 1987] A. L. Besse, Einstein manifolds, Ergebnisse der Mathematik und ihrer Grenzgebiete (3) 10, Springer, Berlin, 1987. MR 88f:53087 Zbl 0613.53001

[Borel 1953a] A. Borel, "La cohomologie mod 2 de certains espaces homogènes", Comment. Math. Helv. 27 (1953), 165-197. MR 15,244h Zbl 0052.40301

[Borel 1953b] A. Borel, "Les bouts des espaces homogènes de groupes de Lie", Ann. of Math. (2) 58 (1953), 443-457. MR 15,199c Zbl 0053.13002

[Borel and Hirzebruch 1958] A. Borel and F. Hirzebruch, "Characteristic classes and homogeneous spaces. I", Amer. J. Math. 80 (1958), 458-538. MR 21 \#1586 Zbl 0097.36401

[Borel and Serre 1953] A. Borel and J.-P. Serre, "Groupes de Lie et puissances réduites de Steenrod", Amer. J. Math. 75 (1953), 409-448. MR 15,338b Zbl 0050.39603

[Bott 1969] R. Bott, Lectures on $K(X)$, W. A. Benjamin, New York-Amsterdam, 1969. MR 41 \#2667 Zbl 0194.23904

[Deligne et al. 1975] P. Deligne, P. Griffiths, J. Morgan, and D. Sullivan, "Real homotopy theory of Kähler manifolds”, Invent. Math. 29:3 (1975), 245-274. MR 52 \#3584 Zbl 0312.55011

[Eschenburg 1992a] J.-H. Eschenburg, “Cohomology of biquotients”, Manuscr. Math. 75:2 (1992), 151-166. MR 93e:57070 Zbl 0769.53029

[Eschenburg 1992b] J.-H. Eschenburg, "Inhomogeneous spaces of positive curvature", Differential Geom. Appl. 2:2 (1992), 123-132. MR 94j:53044 Zbl 0778.53033

[Gilmore 1967] M. E. Gilmore, "Complex Stiefel manifolds, some homotopy groups and vector fields”, Bull. Amer. Math. Soc. 73 (1967), 630-633. MR 35 \#4919 Zbl 0158.42201

[Grove and Ziller 2008] K. Grove and W. Ziller, "Lifting group actions and nonnegative curvature", preprint, 2008. arXiv 0801.0767

[Hsiang and Su 1968] W.-y. Hsiang and J. C. Su, "On the classification of transitive effective actions on Stiefel manifolds", Trans. Amer. Math. Soc. 130 (1968), 322-336. MR 36 \#4581 Zbl 0199.27104

[Husemoller 1975] D. Husemoller, Fibre bundles, 2nd ed., Graduate Texts in Mathematics 20, Springer, New York, 1975. MR 51 \#6805 Zbl 0307.55015

[James 1971] I. M. James, "On the homotopy type of Stiefel manifolds", Proc. Amer. Math. Soc. 29 (1971), 151-158. MR 43 \#1184 Zbl 0217.19901

[James and Whitehead 1954] I. M. James and J. H. C. Whitehead, "The homotopy theory of sphere bundles over spheres. I", Proc. London Math. Soc. (3) 4 (1954), 196-218. MR 15,892b Zbl 0056. 16703 
[Kobayashi and Nomizu 1963] S. Kobayashi and K. Nomizu, Foundations of differential geometry, vol. 1, Wiley, New York-London, 1963. MR 27 \#2945 Zbl 0119.37502

[Kotschick 2001] D. Kotschick, "On products of harmonic forms", Duke Math. J. 107:3 (2001), 521-531. MR 2002c:53076 Zbl 1036.53030

[Kotschick and Terzić 2003] D. Kotschick and S. Terzić, "On formality of generalized symmetric spaces”, Math. Proc. Cambridge Philos. Soc. 134:3 (2003), 491-505. MR 2004c:53071 Zbl 1042. 53035

[Kotschick and Terzić 2009] D. Kotschick and S. Terzić, "Chern numbers and the geometry of partial flag manifolds", Comment. Math. Helv. 84:3 (2009), 587-616. MR 2010i:53089 Zbl 1169.53039

[Kramer 2002] L. Kramer, Homogeneous spaces, Tits buildings, and isoparametric hypersurfaces, Mem. Amer. Math. Soc. 752, American Mathematical Society, Providence, RI, 2002. MR 2003m: 53078 Zbl 1027.57002

[Kuin' 1968] D. Kuin', “The Poincaré polynomials of compact homogeneous Riemannian spaces with irreducible stationary group”, Trudy Sem. Vektor. Tenzor. Anal. 14 (1968), 33-93. In Russian. MR 43 \#427

[Lundell 1992] A. T. Lundell, "Concise tables of James numbers and some homotopy of classical Lie groups and associated homogeneous spaces", pp. 250-272 in Algebraic topology (San Feliu de Guíxols, 1990), edited by J. Aguadé et al., Lecture Notes in Math. 1509, Springer, Berlin, 1992. MR 93g:55019 Zbl 0752.57020

[Mimura 1967] M. Mimura, "The homotopy groups of Lie groups of low rank", J. Math. Kyoto Univ. 6 (1967), 131-176. MR 34 \#6774 Zbl 0171.44101

[Ōguchi 1969] K. Ōguchi, "Homotopy groups of $\operatorname{Sp}(n) / \mathrm{Sp}(n-2)$ ", J. Fac. Sci. Univ. Tokyo Sect. I 16 (1969), 179-201. MR 41 \#4542 Zbl 0191.53803

[Onishchik 1963] A. L. Onishchik, "Transitive compact transformation groups", Mat. Sb., N. Ser. 60:102 (1963), 447-485. In Russian; translated in Amer. Math. Soc. Transl. 55 (1966), 153-194. Zbl 0203.26302

[Onishchik 1995] A. L. Onishchik, Topologiya tranzitivnykh grupp preobrazovanii, Fizmatlit "Nauka”, Moscow, 1995. In Russian. MR 97j:57057 Zbl 0841.57001

[Sullivan 1975] D. Sullivan, "Differential forms and the topology of manifolds", pp. 37-49 in Manifolds (Tokyo, 1973), edited by A. Hattori, Univ. Tokyo Press, Tokyo, 1975. MR 51 \#6838 Zbl 0319.58005

[Takeuchi 1962] M. Takeuchi, "On Pontrjagin classes of compact symmetric spaces”, J. Fac. Sci. Univ. Tokyo Sect. I 9 (1962), 313-328 (1962). MR 26 \#2548 Zbl 0108.35802

[Terzić 2003] S. Terzić, "Rational homotopy groups of generalised symmetric spaces", Math. Z. 243:3 (2003), 491-523. MR 2004k:55011 Zbl 1021.55005

[Terzich 2001] S. Terzich, "Real cohomology of generalized symmetric spaces", Fundam. Prikl. Mat. 7:1 (2001), 131-157. In Russian. MR 2002h:22029

[Totaro 2003] B. Totaro, "Curvature, diameter, and quotient manifolds", Math. Res. Lett. 10:2-3 (2003), 191-203. MR 2004m:53067 Zbl 1063.53038

[Wang and Ziller 1993] M. Wang and W. Ziller, "Symmetric spaces and strongly isotropy irreducible spaces", Math. Ann. 296:2 (1993), 285-326. MR 94g:53042 Zbl 0804.53075

[Wolf and Gray 1968a] J. A. Wolf and A. Gray, "Homogeneous spaces defined by Lie group automorphisms, I”, J. Differential Geometry 2 (1968), 77-114. MR 38 \#4625a Zbl 0169.24103

[Wolf and Gray 1968b] J. A. Wolf and A. Gray, "Homogeneous spaces defined by Lie group automorphisms, II”, J. Differential Geometry 2 (1968), 115-159. MR 38 \#4625b Zbl 0182.24702 
Received September 17, 2009.

D. KOTSCHICK

MATHEMATISCHES Institut

LMU MÜNCHEN

THERESIENSTR. 39

80333 MÜNCHEN

GERMANY

dieter@member.ams.org

S. TERZIĆ

FACULTY OF SCIENCE

UNIVERSITY OF MONTENEGRO

DŽORDŽA VAŠINGTONA BB

81000 PODGORICA

Montenegro

sterzic@ac.me 


\title{
PACIFIC JOURNAL OF MATHEMATICS
}

\author{
http://www.pjmath.org \\ Founded in 1951 by \\ E. F. Beckenbach (1906-1982) and F. Wolf (1904-1989)
}

\section{EDITORS}

V. S. Varadarajan (Managing Editor)

Department of Mathematics

University of California

Los Angeles, CA 90095-1555

pacific@math.ucla.edu

Vyjayanthi Chari

Department of Mathematics

University of California

Riverside, CA 92521-0135

chari@math.ucr.edu

Robert Finn

Department of Mathematics Stanford University

Stanford, CA 94305-2125

finn@math.stanford.edu

Kefeng Liu

Department of Mathematics

University of California

Los Angeles, CA 90095-1555

liu@math.ucla.edu
Darren Long

Department of Mathematics

University of California

Santa Barbara, CA 93106-3080

long@math.ucsb.edu

Jiang-Hua Lu

Department of Mathematics

The University of Hong Kong

Pokfulam Rd., Hong Kong jhlu@maths.hku.hk

Alexander Merkurjev

Department of Mathematics

University of California

Los Angeles, CA 90095-1555

merkurev@math.ucla.edu
Sorin Popa

Department of Mathematics University of California

Los Angeles, CA 90095-1555 popa@math.ucla.edu

Jie Qing

Department of Mathematics

University of California

Santa Cruz, CA 95064

qing@cats.ucsc.edu

Jonathan Rogawski

Department of Mathematics

University of California

Los Angeles, CA 90095-1555

jonr@math.ucla.edu

\section{PRODUCTION}

pacific@math.berkeley.edu

\begin{abstract}
Silvio Levy, Scientific Editor Matthew Cargo, Senior Production Editor
\end{abstract}
ACADEMIA SINICA, TAIPEI

CALIFORNIA INST. OF TECHNOLOGY

INST. DE MATEMÁTICA PURA E APLICADA

KEIO UNIVERSITY

MATH. SCIENCES RESEARCH INSTITUTE

NEW MEXICO STATE UNIV.

OREGON STATE UNIV.

\section{SUPPORTING INSTITUTIONS}

STANFORD UNIVERSITY
UNIV. OF BRITISH COLUMBIA
UNIV. OF CALIFORNIA, BERKELEY
UNIV. OF CALIFORNIA, DAVIS
UNIV. OF CALIFORNIA, LOS ANGELES
UNIV. OF CALIFORNIA, RIVERSIDE
UNIV. OF CALIFORNIA, SAN DIEGO
UNIV. OF CALIF., SANTA BARBARA

UNIV. OF CALIF., SANTA CRUZ

UNIV. OF MONTANA

UNIV. OF OREGON

UNIV. OF SOUTHERN CALIFORNIA

UNIV. OF UTAH

UNIV. OF WASHINGTON

WASHINGTON STATE UNIVERSITY

These supporting institutions contribute to the cost of publication of this Journal, but they are not owners or publishers and have no responsibility for its contents or policies.

See inside back cover or www.pjmath.org for submission instructions.

The subscription price for 2011 is US \$420/year for the electronic version, and \$485/year for print and electronic.

Subscriptions, requests for back issues from the last three years and changes of subscribers address should be sent to Pacific Journal of Mathematics, P.O. Box 4163, Berkeley, CA 94704-0163, U.S.A. Prior back issues are obtainable from Periodicals Service Company, 11 Main Street, Germantown, NY 12526-5635. The Pacific Journal of Mathematics is indexed by Mathematical Reviews, Zentralblatt MATH, PASCAL CNRS Index, Referativnyi Zhurnal, Current Mathematical Publications and the Science Citation Index.

The Pacific Journal of Mathematics (ISSN 0030-8730) at the University of California, c/o Department of Mathematics, 969 Evans Hall, Berkeley, CA 94720-3840, is published monthly except July and August. Periodical rate postage paid at Berkeley, CA 94704, and additional mailing offices. POSTMASTER: send address changes to Pacific Journal of Mathematics, P.O. Box 4163, Berkeley, CA 94704-0163.

PJM peer review and production are managed by EditFLOW ${ }^{\mathrm{TM}}$ from Mathematical Sciences Publishers.

PUBLISHED BY PACIFIC JOURNAL OF MATHEMATICS

at the University of California, Berkeley 94720-3840

A NON-PROFIT CORPORATION

Typeset in LATEX

Copyright $(02011$ by Pacific Journal of Mathematics 


\section{PACIFIC JOURNAL OF MATHEMATICS}

Volume $249 \quad$ No. $1 \quad$ January 2011

Metabelian SL $(n, \mathbb{C})$ representations of knot groups, II: Fixed points

HANS U. BODEN and STEFAN FRIEDL

Lewis-Zagier correspondence for higher-order forms

ANTON DEITMAR

Topology of positively curved 8-dimensional manifolds with symmetry

ANAND DESSAI

Strong Kähler with torsion structures from almost contact manifolds

MARISA FERnÁNDEZ, ANNA FINO, LUIS UGARTE and RAQUEL

VILLACAMPA

Connections between Floer-type invariants and Morse-type invariants of Legendrian knots

MICHAEL B. HENRY

A functional calculus for unbounded generalized scalar operators on Banach spaces

DRAGOLJUB KEČKIĆ and ĐORĐE KRTINIĆ

Geometric formality of homogeneous spaces and of biquotients

D. KoTSCHICK and S. TERZIĆ

Positive solutions for a nonlinear third order multipoint boundary value problem

Yang LiU, Zhang Weiguo, Liu Xiping, Shen Chunfang and Chen HUA

The braid group surjects onto $G_{2}$ tensor space

SCOTT MORRISON

Analogues of the Wiener Tauberian and Schwartz theorems for radial functions on symmetric spaces

E. K. NARAyAnAn and Alladi Sitaram

Semidirect products of representations up to homotopy

Yunhe SHENG and Chenchang ZHU

Homology sequence and excision theorem for Euler class group

YONG YANG 